\title{
POLYNOMIAL DIFFERENTIAL EQUATIONS WITH MANY REAL OVALS IN THE SAME ALGEBRAIC COMPLEX SOLUTION
}

\author{
A. Lins Neto
}

\begin{abstract}
Let $\mathrm{Fol}_{\mathbb{R}}(2, d)$ be the space of real algebraic foliations of degree $d$ in $\mathbb{R P}(2)$. For fixed $d$, let $\operatorname{Int}_{\mathbb{R}}(2, d)=\left\{\mathcal{F} \in \mathrm{Fol}_{\mathbb{R}}(2, d) \mid \mathcal{F}\right.$ has a non-constant rational first integral $\}$. Given $\mathcal{F} \in \operatorname{Int}_{\mathbb{R}}(2, d)$, with primitive first integral $G$, set $O(\mathcal{F})=$ number of real ovals of the generic level $(G=c)$. Let $O(d)=\sup \left\{O(\mathcal{F}) \mid \mathcal{F} \in \operatorname{Int}_{\mathbb{R}}(2, d)\right\}$.

The main purpose of this paper is to prove that $O(d)=+\infty$ for all $d \geq 5$.
\end{abstract}

\section{Contents}

1. Introduction 379

2. Some properties of the family $\mathbb{F}_{4}$

3. Pulling-back the family $\mathbb{F}_{4} \quad 390$

4. Other families 395

References 398

\section{Introduction}

It is well known (Harnack's theorem) that a real smooth algebraic curve of degree $d$ in $\mathbb{R P}(2)$ has at most $\frac{(d-1)(d-2)}{2}+1$ connected components (ovals). A similar question in the context of real algebraic foliations can be posed. Let $\mathrm{Fol}_{\mathbb{R}}(2, d)$ be the set of algebraic foliations in $\mathbb{R P}(2)$ of degree $d$. A foliation $\mathcal{F} \in \operatorname{Fol}_{\mathbb{R}}(2, d)$ can be complexified to one in $\mathbb{C P}(2)$ that we denote $\mathcal{F}_{\mathbb{C}}$. We will study the following problem:

2010 Mathematics Subject Classification. 34C09, 34C08, 37F75.

Key words. Real ovals, differential equations.

This research was partially supported by Pronex. 
Problem 1. Given $d \geq 2$ does there exists $N(d) \in \mathbb{N}$ such that for any complex algebraic leaf $L$ of a foliation $\mathcal{F}_{\mathbb{C}}, \mathcal{F} \in \operatorname{Fol}_{\mathbb{R}}(2, d)$, then the number of ovals of $L \cap \mathbb{R} \mathbb{P}(2)$ is $\leq N(d)$ ?

We would like to remark that for $d=1$ there exists such a bound: $N(1)=1$.

The main purpose of this paper is to prove that there is no such a bound for all $d \geq 5$. In order to pose the main result, let us recall some facts and fix some notations concerning the subject.

Let $\mathrm{Fol}(2, d)$ be the space of holomorphic foliations of degree $d$ in $\mathbb{C P}(2)$. Any foliation $\mathcal{F} \in \mathrm{Fol}(2, d)$ can be represented in homogeneous coordinates by an integrable 1 -form

$$
\Omega_{\mathcal{F}}=P(x, y, z) d x+Q(x, y, z) d y+R(x, y, z) d z
$$

where $P, Q$ and $R$ are homogeneous polynomials of degree $d+1$ and $x \cdot P+y \cdot Q+z \cdot R \equiv 0$ (cf. $[\mathbf{L N}])$. Geometrically the degree of $\mathcal{F}$ is the number of tangencies of $\mathcal{F}$ with a generic straight line of $\mathbb{C P}(2)$. For instance, in the affine coordinates system $(z=1)$ the foliation is defined by the differential equation $\omega=0$, where

$$
\omega=P(x, y, 1) d x+Q(x, y, 1) d y,
$$

and the number of tangencies of $\mathcal{F}$ in $\mathbb{C}^{2}=(z=1) \subset \mathbb{C P}(2)$ with the line $(y=a x+b)$ is the number of complex solutions of the equation $f(x)=0$, where $f(x)=P(x, a x+b)-a Q(x, a x+b)$. With the condition $x \cdot P+y \cdot Q+z \cdot R=0$ and if $a, b \in \mathbb{C}$ are generic then the polynomial $f(x)$ has degree $d$.

We will denote by $\operatorname{sing}(\mathcal{F})$ the singular set of $\mathcal{F}$. If $\Omega_{\mathcal{F}}$ is as in (1) then in homogeneous coordinates we have $\operatorname{sing}(\mathcal{F})=(P=Q=R=0)$.

The set of real algebraic foliations of degree $d$, in these coordinates, is

$$
\begin{aligned}
\mathrm{Fol}_{\mathbb{R}}(2, d)=\left\{\mathcal{F} \mid \Omega_{\mathcal{F}}=P d x+Q d y+R d z,\right. \\
x . P+y \cdot Q+z \cdot R=0, \quad P, Q, R \in \mathbb{R}[x, y, z] \\
\quad \text { and } P, Q \text { and } R \text { are homogeneous of degree } d+1\} .
\end{aligned}
$$

From now on, we will suppose the homogeneous coordinates fixed.

Let $\operatorname{Int}(2, d)=\{\mathcal{F} \in \operatorname{Fol}(2, d) \mid \mathcal{F}$ has a non-constant rational first integral $\}$ and $\operatorname{Int}_{\mathbb{R}}(2, d)=\operatorname{Int}(2, d) \cap \mathrm{Fol}_{\mathbb{R}}(2, d)$. It is well known that if $\mathcal{F} \in \operatorname{Fol}_{\mathbb{R}}(2, d)$ has a non-constant rational first integral then it has one, say $F / G$, where $F, G \in \mathbb{R}[x, y, z]$, that is with real coefficients. For a fixed $\mathcal{F} \in \operatorname{Int}_{\mathbb{R}}(2, d)$ we will denote by $O(\mathcal{F})$ the number of ovals of the 
generic level $\left(G_{\mathcal{F}}=c\right), c \in \mathbb{R}$, where $G_{\mathcal{F}}$ is a real primitive rational first integral of $\mathcal{F}$.

The main goal of this paper is to prove the following result:

Theorem 1. For all $d \geq 5$ there are families $\left(\mathcal{F}_{\alpha}\right)_{\alpha \in J}, J=(a<t<$ b) $\subset \mathbb{R}$, in $\mathrm{Fol}_{\mathbb{R}}(2, d)$ with the following properties:

(P.1) $\mathcal{F}_{\alpha} \in \operatorname{Int}_{\mathbb{R}}(2, d)$ if, and only if, $\alpha \in \mathbb{Q} \cap J$.

(P.2) The set $\left\{O\left(\mathcal{F}_{\alpha}\right) \mid \alpha \in \mathbb{Q} \cap J\right\}$ is unbounded.

(P.3) If $\alpha \notin \mathbb{Q} \cap J$ then for almost all complex leaves $L$ of the complexification of $\mathcal{F}_{\alpha}$ such that $L \cap \mathbb{R P}(2) \neq \emptyset$ then $L \cap \mathbb{R} \mathbb{P}(2)$ has an infinite number of connected components.

In particular, (P.2) implies that for all $d \geq 5$ we have

$$
\sup \left\{O(\mathcal{F}) \mid \mathcal{F} \in \operatorname{Int}_{\mathbb{R}}(2, d)\right\}=+\infty .
$$

The proof of Theorem 1 will be based in $[\mathbf{L N}]$ and in some results of $[\mathbf{L N}-\mathbf{1}]$. In $[\mathbf{L N}]$, for any degree $d \geq 2$, we give examples of 1-parameter families of foliations $\mathbb{F}_{d}=\left(\mathcal{F}_{\alpha}^{d}\right)_{\alpha \in \overline{\mathbb{C}}}$ in $\operatorname{Fol}(2, d)$, with the following properties:

(I) The set $E_{d}=\left\{\alpha \in \overline{\mathbb{C}} \mid \mathcal{F}_{\alpha}^{d}\right.$ has a non-constant rational first integral $\}$ is countable and dense in $\overline{\mathbb{C}}$. Denote by $G_{\alpha}^{d}$ a primitive rational first integral of $\mathcal{F}_{\alpha}^{d}$.

(II) The set $\left\{\operatorname{dg}\left(G_{\alpha}^{d}\right) \mid \alpha \in E_{d}\right\}$ is unbounded (dg = degree).

(III) The family is generically equisingular, in the following sense:

(a) There exists a finite subset $F$ of $\overline{\mathbb{C}}$ such that for any $\alpha \in \overline{\mathbb{C}} \backslash F$ the singularities of $\mathcal{F}_{\alpha}^{d}$ are non-degenerate.

(b) For any fixed $\alpha_{o} \notin F$ and any singularity $p_{o}$ of $\mathcal{F}_{\alpha_{o}}^{d}$ there exists a holomorphic function $p(\alpha)$ defined in a neighborhood of $\alpha_{o}$ such that $p\left(\alpha_{o}\right)=p_{o}, p(\alpha) \in \operatorname{sing}\left(\mathcal{F}_{\alpha}^{d}\right)$ and the germs of $\mathcal{F}_{o}^{d}$ and $\mathcal{F}_{\alpha}^{d}$ at $p_{o}$ and $p(\alpha)$, respectively, are holomorphically equivalent.

The families of degrees $d=2,3,4$ are exhibited explicitly in [LN]. For instance, $\mathbb{F}_{4}$ is defined in affine coordinates by the family of polynomial 1-forms on $\mathbb{C}^{2} \subset \mathbb{C P}(2),\left(\omega_{\alpha}:=\omega-\alpha \cdot \omega_{\infty}\right)_{\alpha \in \overline{\mathbb{C}}}$, where

$$
\left\{\begin{array}{l}
\omega=\left(x^{3}-1\right) x d y-\left(y^{3}-1\right) y d x \\
\omega_{\infty}=\left(x^{3}-1\right) y^{2} d y-\left(y^{3}-1\right) x^{2} d x
\end{array} .\right.
$$


It is shown in $[\mathbf{L N}]$ that for $d \in\{2,3,4\}$ and $\alpha \in E_{d}$ the normalization of a generic level $\left(G_{\alpha}^{d}=c\right)$ is an elliptic curve biholomorphic to $\mathbb{C} /\left\langle 1, e^{2 \pi i / 3}\right\rangle$, where $\langle 1, a\rangle$ denotes the lattice $\{m+n . a \mid m, n \in \mathbb{Z}\}$. In particular, these families cannot satisfy condition (P.2) of Theorem 1. However, for $d \geq 5$ the family $\mathbb{F}_{d}$ is obtained by pulling-back one of the families $\mathbb{F}_{2}, \mathbb{F}_{3}$ or $\mathbb{F}_{4}$ by some fixed rational map $\Phi: \mathbb{C P}(2) \rightarrow \mathbb{C P}(2)$, that is $\mathbb{F}_{d}=\Phi^{*}\left(\mathbb{F}_{j}\right)$, for some $j \in\{2,3,4\}$. In this way, for $d \geq 5$, it is shown in $[\mathbf{L N}]$ that $\mathbb{F}_{d}$ satisfies:

(IV) For a fixed $\alpha \in E_{d}$ denote by $g(\alpha)$ the genus of the generic level $\left(G_{\alpha}^{d}=c\right)$. If $d \geq 5$ then the set $\left\{g(\alpha) \mid \alpha \in E_{d}\right\}$ is unbounded.

As we will see in Section 3, when we pull-back the family $\mathbb{F}_{4}$ by an appropriate rational map $\Phi$ with real coefficients then we get a real family of degree $d=8$ satisfying (P.1), (P.2), and (P.3) of Theorem 1. In Section 4 we will sketch how to obtain families of any degree $d \geq 5$ satisfying (P.1), (P.2) and (P.3).

We would like to observe that property (P.1) will be a consequence of the following result of $[\mathbf{L N}-\mathbf{1}]$ :

(V) For all $d \geq 2$ we have

$$
E_{d}=\left\{a+b \cdot e^{2 \pi i / 3} \mid a, b \in \mathbb{Q}\right\} \cup\{\infty\} .
$$

In particular, $E_{d} \cap \mathbb{R}=\mathbb{Q}$.

Remark 1.1. In [LN-1] it is exhibited another family of degree three such that the set of parameters for which the correspondent foliation has a first integral is $\{a+b . i \mid a, b \in \mathbb{Q}\} \cup\{\infty\}, i=\sqrt{-1}$. Families satisfying properties (P.1), (P.2) and (P.3) of Theorem 1 can be also constructed by pulling-back this particular one.

Theorem 1 motivates the following problems:

Problem 2. Is the statement of Theorem 1 true for degrees $d=2, d=3$ or $d=4$ ?

Problem 3. For an algebraic curve $L \subset \mathbb{C P}(2)$ denote by $O(L)$ the number of connected components of $L \cap \mathbb{R} \mathbb{P}(2)$. Given $\mathcal{F} \in \mathrm{Fol}_{\mathbb{R}}(2, d)$ set

$$
O(\mathcal{F})=\max \{O(L) \mid L \text { is an algebraic leaf of } \mathcal{F}\} .
$$

We would like to observe that an algebraic leaf is automatically irreducible (by the definition of leaf).

A natural question is the following: does there exists $d \geq 2$ such that

$$
\sup \left\{O(\mathcal{F}) \mid \mathcal{F} \in \operatorname{Fol}_{\mathbb{R}}(2, d) \backslash \operatorname{Int}_{\mathbb{R}}(2, d)\right\}<+\infty ?
$$


Remark 1.2. Concerning Problem 3 the following result was proved in $[\mathbf{C}]$ by M. M. Carnicer: let $\mathcal{F}$ be a foliation of degree $d$ in $\mathbb{C P}(2)$ without dicritical singularities. If $L$ is an algebraic leaf of $\mathcal{F}$ then $\operatorname{dg}(L) \leq d+2$. In particular, if $\mathcal{F} \in \operatorname{Fol}_{\mathbb{R}}(2, d)$ then $L \cap \mathbb{R P}(2)$ has at most $\frac{d(d+1)}{2}+1$ ovals.

Remark 1.3. In this remark we consider families of logarithmic foliations in $\operatorname{Fol}_{\mathbb{R}}(2, d)$ from the point of view of Problem 1 . Let $f_{1}, \ldots, f_{r}$ be real irreducible polynomials in two variables, two by two relatively primes, and consider the family of foliations in $\mathbb{R P}(2)$ defined in an affine coordinates system by the $(r-1)$-parametric differential equation $\omega_{\lambda}=0$, where

$$
\omega_{\lambda}=f_{1} \ldots f_{r} \sum_{j=1}^{r} \lambda_{j} \frac{d f_{j}}{f_{j}}, \quad \sum_{j=1}^{r} \lambda_{j} \cdot \operatorname{dg}\left(f_{j}\right)=0 .
$$

Denote by $\mathcal{G}_{\lambda}$ the foliation in $\mathbb{R P}(2)$ defined by $\omega_{\lambda}=0$. If $\lambda \neq$ $(0, \ldots, 0)$, it can be shown that $\mathcal{G}_{\lambda}$ has degree $d$, where $d=\operatorname{dg}\left(f_{1}\right)+$ $\cdots+\operatorname{dg}\left(f_{r}\right)-2$. Moreover, $\mathcal{G}_{\lambda} \in \operatorname{Int}_{\mathbb{R}}(2, d)$ if, and only if $\lambda=c . \mu$, where $\mu \in \mathbb{Z}^{r}$. If $\lambda=\left(n_{1}, \ldots, n_{r}\right) \in \mathbb{Z}^{r}$ and $\operatorname{gcd}\left(n_{1}, \ldots, n_{r}\right)=1$ then a primitive first integral of $\mathcal{G}_{\lambda}$ is $F_{\lambda}=f_{1}^{n_{1}} \ldots f_{r}^{n_{r}}$. In particular, the set

$$
\left\{\operatorname{dg}\left(F_{\lambda}\right) \mid \lambda \in \mathbb{Z}^{r}\right\}
$$

is unbounded. On the other hand, this family does not satisfy property (P.2) of Theorem 1. This is a consequence of the results of Khovanskil in $[\mathbf{K h}]$ wich assert that the maximal number of ovals of the level $\left(F_{\lambda}=c\right)$ has a bound $N\left(f_{1}, \ldots, f_{r}\right)$, which does not depends on $\lambda$, but only on the number of non-zero monomials of $f_{1}, \ldots, f_{r}$. This fact, of course, implies that the maximal number of ovals that can be obtained from a solution of an equation like in (3) inducing a foliation of degree $d$ has a bound which depends only of $d$.

I would like to thank Jean Jacques Risler who convinced me of the importance of the subject and strongly stimulated me to write this paper.

\section{Some properties of the family $\mathbb{F}_{4}$}

We begin this section by describing some properties of the family $\mathbb{F}_{4}$ that will be needed (see $[\mathbf{L N}]$ and $[\mathbf{L N}-1]$ ). We will use the notation $j=$ $e^{2 \pi i / 3}$.

Let $\mathcal{F}_{\alpha}^{4}$ be the foliation defined in the affine coordinates $(x, y) \in \mathbb{C}^{2} \subset$ $\mathbb{C P}(2)$ by $\omega_{\alpha}=\omega-\alpha \cdot \omega_{\infty}$, where $\omega$ and $\omega_{\infty}$ are as in $(2)$. We will consider 
also $\mathcal{F}_{\alpha}^{4}$ defined by the dual vector field $X_{\alpha}$ of $\omega_{\alpha}: X_{\alpha}=X-\alpha . X_{\infty}$, where $X=\left(x^{3}-1\right) x \partial_{x}+\left(y^{3}-1\right) y \partial_{y}$ and $X_{\infty}=\left(x^{3}-1\right) y^{2} \partial_{x}+\left(y^{3}-1\right) x^{2} \partial_{y}$.

The tangency divisor of $\mathcal{F}_{0}^{4}$ and $\mathcal{F}_{\infty}^{4}$, denoted by $\mathcal{L}$, is the union of 9 lines in $\mathbb{C P}(2)$, say $\ell_{1}, \ldots, \ell_{9}$, defined in $\mathbb{C}^{2}$ by

$$
\left(x^{3}-1\right)\left(y^{3}-1\right)\left(y^{3}-x^{3}\right)=0 .
$$

These lines are invariant by all foliations in the family. They intersect two by two in the 12 points of the set

$$
\mathcal{R}=\left\{(a, b) \in \mathbb{C}^{2} \mid a, b \in\left\{1, j, j^{2}\right\}\right\} \cup\{[0: 1: 0],[1: 0: 0]\} .
$$

The points $[0: 1: 0]$ and $[1: 0: 0]$ are contained in the line at infinity of $\mathbb{C}^{2} \subset \mathbb{C P}(2)$. For all $\alpha \in \overline{\mathbb{C}}$ we have $\mathcal{R} \subset \operatorname{sing}\left(\mathcal{F}_{\alpha}^{4}\right)$.

Let us describe $\mathcal{F}_{\alpha}^{4}$ for $\alpha \notin\left\{1, j, j^{2}, \infty\right\}$. In this case, the foliation $\mathcal{F}_{\alpha}^{4}$ has 21 singularities, the 12 in the set $\mathcal{R}$ and 9 more in the set

$\mathcal{S}_{\alpha}=\left(x^{3}-1=y-\alpha x^{2}=0\right) \cup\left(y^{3}-1=x-\alpha y^{2}=0\right) \cup\left(y^{3}-x^{3}=x-\alpha y^{2}=0\right)$.

In particular, each line of $\mathcal{L}$ contains 4 singularities of $\mathcal{F}_{\alpha}^{4}$. For instance, the line $(y=x)$ contains the singularities $(1,1),(j, j),\left(j^{2}, j^{2}\right)$ and $(1 / \alpha, 1 / \alpha)$. The 12 points in $\mathcal{R}$ are of radial type for $\mathcal{F}_{\alpha}^{4}$ : if $p \in \mathcal{R}$ then there exists a local chart $(u, v)$ around $p$ with $u(p)=v(p)=0$ such that $X_{\alpha}=a\left(u \partial_{u}+v \partial_{v}\right), a \neq 0$. In particular, $v / u$ is a local meromorphic first integral of $\mathcal{F}_{\alpha}^{4}$. The 9 points in $\mathcal{S}_{\alpha}$ are of saddle type: if $q \in \mathcal{S}_{\alpha} \cap \ell_{k}$, $k \in\{1, \ldots, 9\}$, then there exists a local chart $(W,(u, v))$ around $q$ such that $u(q)=v(q)=0, \ell_{k} \cap W=(v=0)$ and $X_{\alpha}=a\left(u \partial_{u}-3 v \partial_{v}\right), a \neq 0$. In particular, $u^{3} \cdot v$ is a local holomorphic first integral of $\mathcal{F}_{\alpha}^{4}$.

The resolution of $\mathcal{F}_{\alpha}^{4}$, in the sense of Seidenberg (cf. [ $\left.\mathbf{S e}\right]$ or $[\mathbf{B r}]$ ), is done by blowing-up in the 12 points of $\mathcal{R}$. Denote by $\pi: M \rightarrow \mathbb{C P}(2)$ this blowing-up procedure and set $\tilde{\mathcal{F}}_{\alpha}:=\pi^{*}\left(\mathcal{F}_{\alpha}^{4}\right)$. Let $\tilde{\ell}_{k}$ denote the strict transform of $\ell_{k}$ by $\pi, k=1, \ldots, 9$, and set $\tilde{\mathcal{L}}=\cup_{k=1}^{9} \tilde{\ell}_{k}$. The following properties are proved in $[\mathbf{L N}]$ :

(I) If $\alpha \neq \beta$ then $\tilde{\mathcal{F}}_{\alpha}$ and $\tilde{\mathcal{F}}_{\beta}$ are transverse outside $\tilde{\mathcal{L}}$.

(II) If $\beta \in\left\{1, j, j^{2}, \infty\right\}$ then the foliation $\tilde{\mathcal{F}}_{\beta}$ has a holomorphic first integral $\tilde{F}_{\beta}: M \rightarrow \mathbb{C P}(1)$. Moreover, $\tilde{F}_{\beta}$ is an elliptic fibration with three singular fibers. For instance, $G(x, y):=\left(y^{3}-1\right) /\left(x^{3}-1\right)$ is a rational first integral of $\mathcal{F}_{\infty}^{4}$ and $\tilde{F}_{\infty}=G \circ \pi: M \rightarrow \mathbb{C P}(1)$. The critical levels of $\tilde{F}_{\infty}$ are $0,1, \infty \in \mathbb{C P}(1)$. After blowingdown the -1-component of one of the critical fibers, it becomes of type $I V$ in Kodaira's classification of critical fibers of elliptic fibrations (cf. $[\mathbf{K}])$. 
Remark 2.1. Set $T_{c}:=\tilde{F}_{\infty}^{-1}(c)$, where $c \neq 0,1, \infty$. We would like to observe that $T_{c}$ is an elliptic curve biholomorphic to $\mathbb{C} / \Gamma$, where $\Gamma=$ $\langle 1, j\rangle:=\{m+n . j \mid m, n \in \mathbb{Z}\}$. This is a consequence of the fact that $G^{-1}(c)=\left(y^{3}-c \cdot x^{3}+c-1=0\right)$ admits the automorphism of period three $(x, y) \mapsto(j . x, j \cdot y)$.

We will denote by $\Pi_{c}: \mathbb{C} \rightarrow T_{c}$ a holomorphic universal covering of $T_{c}$. Since $T_{c} \simeq \mathbb{C} / \Gamma$, given $z \in \mathbb{C}$ we will denote by $z \bmod (\Gamma)$ its equivalence class in $\mathbb{C} / \Gamma$.

Remark 2.2. Since $\tilde{\mathcal{F}}_{\alpha}$ is transverse to $\tilde{\mathcal{F}}_{\infty}$ outside $\tilde{\mathcal{L}}$, we can define a global holonomy representation $\Phi_{\alpha}: \Pi_{1}(\mathbb{C P}(1) \backslash\{0,1, \infty\}, c) \rightarrow \operatorname{Aut}\left(T_{c}\right)$, $c \notin\{0,1, \infty\}$. If we consider appropriate generators $\gamma_{1}$ and $\gamma_{2}$ of $\Pi_{1}(\mathbb{C P}(1) \backslash$ $\{0,1, \infty\}, c)$, then we can write $\Phi_{\alpha}\left(\gamma_{k}\right):=f_{\alpha}^{k}, k=1,2$, as $f_{\alpha}^{k}(z)=$ $\left(j . z+A_{k}(\alpha)\right) \bmod (\Gamma)$, where $A_{k}: \mathbb{C} \rightarrow \mathbb{C} / \Gamma$ is holomorphic, $k=1,2$ (cf. $[\mathbf{L N}])$. In particular, the global holonomy is the sub-group $\left\langle f_{\alpha}^{1}, f_{\alpha}^{2}\right\rangle$ of $\operatorname{Aut}\left(T_{c}\right)$, generated by $f_{\alpha}^{1}$ and $f_{\alpha}^{2}$.

Let $a(\alpha):=(1-j)^{-1} A_{1}(\alpha) \bmod (\Gamma)$ and $h: \mathbb{C} / \Gamma \rightarrow \mathbb{C} / \Gamma$ be defined by $h(z)=(z+a(\alpha)) \bmod (\Gamma)$. Since $f_{\alpha}^{1}$ has a fixed point at $a(\alpha)$ we have $h^{-1} \circ f_{\alpha}^{1} \circ h(z)=j . z \bmod (\Gamma)$ and $h^{1} \circ f_{\alpha}^{2} \circ h(z)=(j . z+A(\alpha)) \bmod (\Gamma)$, where $A(\alpha)=A_{2}(\alpha)-A_{1}(\alpha)$. In particular, the global holonomy group of $\tilde{\mathcal{F}}_{\alpha}$ is conjugated to the group $G_{\alpha}$ generated by $f(z):=j . z \bmod (\Gamma)$ and $f_{\alpha}(z)=(j . z+A(\alpha)) \bmod (\Gamma)$. In $[\mathbf{L N}]$ it is proved that $\alpha \mapsto A(\alpha)$ is non-constant.

In $[\mathbf{L N}-1]$ it is proved that the functions $A_{k}(\alpha), k=1,2$, are affine, that is $A_{k}(\alpha)=B_{k} \cdot \alpha+C_{k} \bmod (\Gamma)$, where $B_{k}, C_{k} \in \mathbb{C}$. Hence, $A(\alpha)$ is also affine and we can write $A(\alpha)=(B . \alpha+C) \bmod (\Gamma)$, where $B \in \mathbb{C}^{*}$ and $C \in \mathbb{C}$.

We are mainly interested in the real foliations $\tilde{\mathcal{F}}_{\alpha}, \alpha \in \mathbb{R}$, and how their real leaves cut the real levels of $\tilde{F}_{\infty}$. Let us denote by $M_{\mathbb{R}}$ the strict transform of $\mathbb{R P}(2)$ by $\pi: M \rightarrow \mathbb{C P}(2)$. Remark that $M_{\mathbb{R}}$ is $\mathbb{R} \mathbb{P}(2)$ blowed-up in four points: the four real points of $\mathcal{R}, q_{1}:=(0,0), q_{2}:=$ $(1,1), q_{3}:=[1: 0: 0]$ and $q_{4}:=[0: 1: 0]$. In particular, $M_{\mathbb{R}}$ is diffeomorphic to the non-orientable surface of Euler characteristic -3 . Let us denote by $S_{c}$ the real trace of $T_{c}, S_{c}=M_{\mathbb{R}} \cap \tilde{F}_{\infty}^{-1}(c), c \in \mathbb{R} \backslash\{0,1\}$.

Lemma 2.1. If $c \in \mathbb{R} \backslash\{0,1\}$ then $S_{c}$ is connected and homeomorphic to the circle $\mathbb{S}^{1}$. Moreover, there exists an universal covering $\Pi_{c}: \mathbb{C} \rightarrow T_{c}$ such that $\Pi_{c}(\mathbb{R})=\Pi_{c}(0 \leq t<1)=S_{c}$. 
Proof: If $c \in \mathbb{R} \backslash\{0,1\}$ then $S_{c}$ is the strict transform of $G^{-1}(c) \cap \mathbb{R} \mathbb{P}(2)$ by $\pi$. On the other hand, $G^{-1}(c)=\left(y^{3}-c \cdot x^{3}+c-1=0\right)$. For each $x \in \mathbb{R}$ there is only one $y \in \mathbb{R}$ such that $y=\left[c \cdot x^{3}+1-c\right]^{1 / 3}$. Therefore, $G^{-1}(c) \cap \mathbb{R}^{2}$ is a graph, and so $G^{-1}(c) \cap \mathbb{R} \mathbb{P}(2)$ is connected and homeomorphic to $\mathbb{S}^{1}$. Since $S_{c}$ is the strict transform of $G^{-1}(c) \cap \mathbb{R} \mathbb{P}(2)$, the same is true for it.

Let

$$
\mathcal{P}(z)=\frac{1}{z^{2}}+\sum_{\omega \in \Gamma^{*}}\left(\frac{1}{(z-\omega)^{2}}-\frac{1}{\omega^{2}}\right), \quad \Gamma^{*}=\Gamma \backslash\{0\},
$$

be the Weierstrass $\mathcal{P}$-function associated to the latice $\Gamma$. The cubic $G^{-1}(c)$ in the Weierstrass normal form can be written as $v^{2}=$ $4\left(u^{3}-C\right)$, where $C=35 \sum_{\omega \in \Gamma^{*}} \frac{1}{\omega^{6}} \in \mathbb{R}_{+}$. It is known that $z \in \mathbb{C} \mapsto$ $\left(\mathcal{P}(z), \mathcal{P}^{\prime}(z)\right)=(u, v)$ parametrizes $G^{-1}(c)$ in the normal form, that is $\left(\mathcal{P}^{\prime}\right)^{2}=4\left(\mathcal{P}^{3}-C\right)$. On the other hand, the projective automorphism

$$
\begin{aligned}
& \left\{\begin{array}{l}
x=\frac{b \cdot B \cdot u}{A \cdot v-1 / 2} \\
y=\frac{b(A \cdot v+1 / 2)}{A \cdot v-1 / 2}
\end{array},\right. \\
& \text { where } b=(1-c)^{1 / 3}, \quad A=1 / \sqrt{48 . C} \quad \text { and } \quad B=1 /(4 . c . C)^{1 / 3}
\end{aligned}
$$

sends $G^{-1}(c)$ to the normal form: $v^{2}=4\left(u^{3}-C\right)$. This implies that $G^{-1}(c)$ in the original affine coordinates system is parametrized by

$$
z \in \mathbb{C} \mapsto\left(\frac{b \cdot B \cdot \mathcal{P}(z)}{A \cdot \mathcal{P}^{\prime}(z)-1 / 2}, \frac{b\left(A \cdot \mathcal{P}^{\prime}(z)+1 / 2\right)}{A \cdot \mathcal{P}^{\prime}(z)-1 / 2}\right):=\Phi_{c}(z)
$$

and $\Phi_{c}: \mathbb{C} \rightarrow G^{-1}(c)$ is an universal covering. If $c \in \mathbb{R}$ then we can take $b, A, B \in \mathbb{R}$, because $C>0$. It follows that $\Phi_{c}(\mathbb{R}) \subset G^{-1}(c) \cap \mathbb{R P}(2)$, because $\mathcal{P}(\mathbb{R}), \mathcal{P}^{\prime}(\mathbb{R}) \subset \mathbb{R}$. Since $G^{-1}(c) \cap \mathbb{R} \mathbb{P}(2)$ has just one irreducible component, we get $\Phi_{c}(\mathbb{R})=G^{-1}(c) \cap \mathbb{R} \mathbb{P}(2)$. On the other hand, $\pi_{c}:=$ $\left.\pi\right|_{T_{c}}: T_{c} \rightarrow G^{-1}(c)$ is a biholomorphism. Hence, $\Pi_{c}:=\pi_{c}^{-1} \circ \Phi_{c}: \mathbb{C} \rightarrow T_{c}$ is an universal covering and $\Pi_{c}(\mathbb{R})=S_{c}$. Since 1 is a period of $\Pi_{c}$, we get $\Pi_{c}(0 \leq t<1)=S_{c}$.

From now on we will fix the fiber $T_{c}:=T, c \in \mathbb{R} \backslash\{0,1\}$, where the global holonomy group is calculated. We will set $S:=S_{c}=T \cap M_{\mathbb{R}}$. 
Corollary 2.1. For any $\alpha \in \mathbb{R}$ there exists an universal covering $\Pi_{\alpha}: \mathbb{C} \rightarrow$ $T$ with the following properties:

(a) The global holonomy group in the fiber $T$ is $G_{\alpha}=\left\langle f, f_{\alpha}\right\rangle$, where $f(z)=j . z \bmod (\Gamma)$ and $f_{\alpha}(z)=(j . z+B . \alpha+C) \bmod (\Gamma)$, with $B \in \mathbb{C}^{*}$ and $C \in \mathbb{C}$.

(b) $\Pi_{\alpha}(\mathbb{R}-a(\alpha))=S$, where $\mathbb{R}-a(\alpha)=\{t-a(\alpha) \mid t \in \mathbb{R}\}$ and $a(\alpha)=(1-j)^{-1} A_{1}(\alpha) \bmod (\Gamma)$ is as before.

In particular, $\Pi_{\alpha}^{-1}(S)=\bigcup_{n \in \mathbb{Z}}(\mathbb{R}-a(\alpha)+n . j)$.

Proof: Consider the universal covering $\Pi_{c}: \mathbb{C} \rightarrow T$ as in Lemma 2.1, for which $\Pi_{c}(\mathbb{R})=S$. The global holonomy group in the fiber $T$ is generated by $f_{\alpha}^{1}$ and $f_{\alpha}^{2}$, which are covered by the maps $\hat{f}_{\alpha}^{k}(z):=j . z+A_{k}(\alpha)$, that is $\Pi_{c} \circ \hat{f}_{\alpha}^{k}(z)=f_{\alpha}^{k}(z) \circ \Pi_{c}, k=1,2$. On the other hand, if $\hat{h}_{\alpha}(z):=z+a(\alpha)$ then $\hat{f}:=\hat{h}_{\alpha}^{-1} \circ \hat{f}_{\alpha}^{1} \circ \hat{h}_{\alpha}$ and $\hat{f}_{\alpha}:=\hat{h}_{\alpha}^{-1} \circ \hat{f}_{\alpha}^{2} \circ \hat{h}_{\alpha}$ are of the form $\hat{f}(z)=j . z$ and $\hat{f}_{\alpha}(z)=j . z+A(\alpha)$, where $A(\alpha)=A_{2}(\alpha)-A_{1}(\alpha)=B . \alpha+C$. Therefore, the universal covering $\Pi_{\alpha}:=\Pi_{c} \circ \hat{h}_{\alpha}$ satisfies $\Pi_{\alpha}(\mathbb{R}-a(\alpha))=$ $S, \Pi_{\alpha} \circ \hat{f}=f_{\alpha}^{1} \circ \Pi_{\alpha}$ and $\Pi_{\alpha} \circ \hat{f}_{\alpha}=f_{\alpha}^{2} \circ \Pi_{\alpha}$.

Recall that $E=\left\{\alpha \in \mathbb{C P}(1) \mid \tilde{\mathcal{F}}_{\alpha}\right.$ has a first integral $\}$. In $[\mathbf{L N}]$ it is proved that the following conditions are equivalent:

(I) $\alpha \in E$.

(II) $G_{\alpha}$ is finite.

(III) $A(\alpha) \in \mathbb{Q} . \Gamma:=\{a+b . j \mid a, b \in \mathbb{Q}\}$.

\section{Notations.}

(a) For $\alpha \in \mathbb{C}$ fixed, set $\Gamma(\alpha)=\{c \cdot A(\alpha) \bmod (\Gamma) \mid c \in \Gamma\}$ and for $\alpha \in \mathbb{R}$, set $\Gamma_{\mathbb{R}}(\alpha)=\Gamma(\alpha) \cap \mathbb{R} \bmod (\Gamma)$.

(b) We have seen in Lemma 2.1 and Corollary 2.1 that $\Pi_{c}(\mathbb{R})=\Pi_{\alpha}(\mathbb{R}-$ $a(\alpha))=S$. We define a segment $I$ on $S$ as the image of an open interval $I:=\Pi_{c}((a, b)) \subset S,(a, b) \subset \mathbb{R}, a<b$.

(c) Given $\alpha \in \mathbb{C} \cup\{\infty\}$ and $q \in M \backslash \operatorname{sing}\left(\tilde{\mathcal{F}}_{\alpha}\right)$ denote by $\ell(\alpha, q)$ the complex leaf of $\tilde{\mathcal{F}}_{\alpha}$ through $q$. If $\alpha \in \mathbb{R} \cup\{\infty\}$ and $q \in M_{\mathbb{R}} \backslash \operatorname{sing}\left(\tilde{\mathcal{F}}_{\alpha}\right)$ then set $\ell_{\mathbb{R}}(\alpha, q):=\ell(\alpha, q) \cap M_{\mathbb{R}}$.

(d) Given $\alpha \in E \cap \mathbb{R}$, a point $q \in M_{\mathbb{R}} \backslash \operatorname{sing}\left(\tilde{\mathcal{F}}_{\alpha}\right)$ and a segment $I$ of $S$ define

$$
N(\alpha, q, I)=\#\left(\ell_{\mathbb{R}}(\alpha, q) \cap I\right) .
$$

Note that $N(\alpha, q, I)$ is always finite, because $\tilde{\mathcal{F}}_{\alpha}$ is transverse to $S$ and has rational first integral for any $\alpha \in E \cap \mathbb{R}$. 
Lemma 2.2. For any fixed $\alpha \in \mathbb{R}, q_{o} \in S$ and $z_{o} \in \mathbb{C}$ such that $\Pi_{\alpha}\left(z_{o}\right)=$ $q_{o}$ we have:

$$
\ell_{\mathbb{R}}\left(\alpha, q_{o}\right) \cap S=\Pi_{\alpha}\left(z_{o}+\Gamma_{\mathbb{R}}(\alpha)\right),
$$

where $z_{o}+\Gamma_{\mathbb{R}}(\alpha)=\left\{z_{o}+t \mid t \in \Gamma_{\mathbb{R}}(\alpha)\right\}$. In particular,

(a) $\ell_{\mathbb{R}}\left(\alpha, q_{o}\right) \cap S$ is dense in $S \Longleftrightarrow \alpha \in \mathbb{R} \backslash \mathbb{Q}$.

(b) Let $\alpha_{o} \in \mathbb{R} \backslash \mathbb{Q}$ and $\left(\alpha_{n}\right)_{n \geq 1}$ be a sequence in $\mathbb{Q}$ such that $\lim _{n \rightarrow \infty} \alpha_{n}=$ $\alpha_{o}$. Then for any segment $I$ of $S$ and any sequence $\left(q_{n}\right)_{n \geq 1}$ in $S$ we have

$$
\lim _{n \rightarrow \infty} N\left(\alpha_{n}, q_{n}, I\right)=+\infty .
$$

Proof: Since $S=\Pi_{\alpha}(\mathbb{R}-a(\alpha))$, we can assume that $z_{o} \in \mathbb{R}-a(\alpha)$.

The global holonomy group $G_{\alpha}$ is constructed in such a way that for any $q \in T$ we have $\ell(\alpha, q) \cap T=\left\{g(q) \mid g \in G_{\alpha}\right\}$. Let $\hat{G}_{\alpha}=\{\hat{g} \in$ Aut $\left.(\mathbb{C}) \mid \Pi_{\alpha} \circ \hat{g}=g \circ \Pi_{\alpha}, g \in G_{\alpha}\right\}$. If $\hat{G}_{\alpha}\left(z_{o}\right)$ denotes the orbit of $z_{o}$ by $\hat{G}_{\alpha}$ then

$$
\ell\left(\alpha, q_{o}\right) \cap T=\Pi_{\alpha}\left(\hat{G}_{\alpha}\left(z_{o}\right)\right) .
$$

Since $S=T \cap M_{\mathbb{R}}=\Pi_{\alpha}(\mathbb{R}-a(\alpha))$ we get

$$
\ell_{\mathbb{R}}\left(\alpha, q_{o}\right) \cap S=\Pi_{\alpha}\left(\hat{G}_{\alpha}\left(z_{o}\right) \cap[\mathbb{R}-a(\alpha)]\right) .
$$

The transformations $\hat{g} \in \hat{G}_{\alpha}$ for which $\hat{g}(\mathbb{R}-a(\alpha))=\mathbb{R}-a(\alpha)$ are in the group $\hat{H}_{\alpha}=\left\{\hat{g} \in \hat{G}_{\alpha} \mid \hat{g}(z)=z+t, t \in \Gamma_{\mathbb{R}}(\alpha)\right\}$. Therefore,

$$
\ell_{\mathbb{R}}\left(\alpha, q_{o}\right) \cap S=\Pi_{\alpha}\left(z_{o}+\Gamma_{\mathbb{R}}(\alpha)\right) .
$$

It follows from (5) that $\ell_{\mathbb{R}}\left(\alpha, q_{o}\right) \cap S$ is dense in $S$ if, and only if, $\Gamma_{\mathbb{R}}(\alpha)$ is dense in $\mathbb{R} \bmod (\Gamma)=\mathbb{R} \bmod (1)$. The next claim implies assertion (a) of Lemma 2.2.

Claim 2.1. The set $\Gamma(\alpha)$ is an additive sub-group of $\mathbb{C} / \Gamma$, whereas if $\alpha \in \mathbb{R}$ then $\Gamma_{\mathbb{R}}(\alpha)$ is an additive sub-group of $\mathbb{R} \bmod (1) \simeq S^{1}$. Moreover, $\Gamma_{R}(\alpha)$ is dense in $\mathbb{R} \bmod (1)$ if, and only if, $\alpha \in \mathbb{R} \backslash \mathbb{Q}$.

Proof: The first two assertions are consequence of the definitions. On the other hand, we will prove at the end that

$$
\Gamma_{\mathbb{R}}(\alpha)=\{\ell \mu(\alpha-1) \bmod (1) \mid \ell \in \mathbb{Z}\},
$$

where $\mu \in \mathbb{Q} \backslash\{0\}$. Note that (6) implies the claim:

$\alpha \in \mathbb{R} \backslash \mathbb{Q} \Longleftrightarrow \mu .(\alpha-1) \in \mathbb{R} \backslash \mathbb{Q} \Longleftrightarrow \Gamma_{\mathbb{R}}(\alpha)$ is dense in $\mathbb{R} \bmod (1)$. 
Recall that $A(\alpha)=(B . \alpha+C) \bmod (\Gamma)$ where $B \in \mathbb{C}^{*}$ and $C \in \mathbb{C}$. We will use the following facts proved in $[\mathbf{L N}]$ :

(i) If $\alpha \in\left\{1, j, j^{2}\right\}$ then $\#\left(G_{\alpha}\right)=3$.

(ii) $\#\left(G_{0}\right)=9$.

(iii) The group $G_{\alpha}$ can be explicitly written as:

$G_{\alpha}=\left\{g \mid g(z \bmod (\Gamma))=(\lambda . z+b . A(\alpha)) \bmod (\Gamma), \lambda \in\left\{1, j, j^{2}\right\}\right.$ and $\left.b \in \Gamma\right\}$.

We can deduce from (i) and (iii) that $A(1)=A(j)=A\left(j^{2}\right)=0 \bmod (\Gamma)$. Now,

$A(1)=0 \bmod (\Gamma) \Longrightarrow B=-C \bmod (\Gamma) \Longrightarrow A(\alpha)=B(\alpha-1) \bmod (\Gamma)$

and

$A(j)=A\left(j^{2}\right)=0 \bmod (\Gamma) \Longrightarrow B(j-1), B\left(j^{2}-1\right) \in \Gamma \Longrightarrow B \in \frac{1}{3} \Gamma$.

It follows from (ii) that $A(0) \notin \Gamma$. Since $A(0)=-B \bmod (\Gamma)$, we get $B \notin \Gamma$.

Therefore, we can write $B=\frac{k}{3}(m+n . j)$, where, $k, m, n \in \mathbb{Z}$ and, either $(m, n)=(1,0)$, or $(m, n)=(0,1)$, or $m, n \neq 0$ and $\operatorname{gcd}(m, n)=1$. Since $B \notin \Gamma$ we must have also $3 \nmid k$ and $3 \nmid k . m$ or $3 \nmid k . n$ if $m, n \neq 0$.

Set $\mu:=\frac{k}{3}\left(m^{2}+n^{2}-m \cdot n\right)=\left(m+n \cdot j^{2}\right) \cdot B \in \mathbb{Q} \backslash\{0\}$ and $X_{\alpha}:=$ $\{\ell \cdot \mu(\alpha-1) \bmod (1) \mid \ell \in \mathbb{Z}\}$ and let us prove that $\Gamma_{\mathbb{R}}(\alpha)=X_{\alpha}$.

First of all recall that

$$
\Gamma_{\mathbb{R}}(\alpha)=\{t \bmod (1) \mid t=c . B(\alpha-1), c \in \Gamma, c . B \in \mathbb{R}\} .
$$

In particular, $\Gamma_{\mathbb{R}}(1)=\{0 \bmod (1)\}=X_{1}$. If $\alpha \in \mathbb{R} \backslash\{1\}$ then $\mu(\alpha-$ 1) $\in \mathbb{R}$. Since $\mu=\left(m+n \cdot j^{2}\right) . B$ we get $\mu(\alpha-1) \bmod (1) \in \Gamma_{\mathbb{R}}(\alpha)$. Hence, $X_{\alpha} \subset \Gamma_{\mathbb{R}}(\alpha)$, because $X_{\alpha}$ and $\Gamma_{\mathbb{R}}(\alpha)$ are additive sub-groups of $\mathbb{R} \bmod (1)$. On the other hand, if c.B. $(\alpha-1) \in \mathbb{R}$ for some $c \in \Gamma$, then

$$
\begin{aligned}
c . B \in \mathbb{R} \Longrightarrow c .(m+n . j) \in \mathbb{R} \cap \Gamma \Longrightarrow c=\ell .\left(m+n . j^{2}\right), \\
\quad \ell \in \mathbb{Z} \Longrightarrow c . B=\ell . \mu, \ell \in \mathbb{Z} \Longrightarrow \Gamma_{\mathbb{R}}(\alpha) \subset X_{\alpha},
\end{aligned}
$$

which proves (6) and the claim.

Proof of (b) of Lemma 2.2: Let $\alpha \in \mathbb{Q} \backslash\{1\}$, so that $\mu$. $(\alpha-1) \in \mathbb{Q} \backslash\{0\}$. Set $\mu .(\alpha-1)=r / s$, where $r, s \in \mathbb{Z}, \operatorname{gcd}(r, s)=1$ and $s>0$. Then there exist $\ell, m \in \mathbb{Z}$ such that $\ell . r-s . m=1$, and so $\ell . r / s=1 / s \bmod (1)$. In this case, we get

$$
\Gamma_{\mathbb{R}}(\alpha)=\left\{\frac{n}{s} \bmod (1) \mid n \in \mathbb{Z}\right\}
$$


Let $(a, b) \subset \mathbb{R}$ be an open interval with $b-a>1 / s$. Then there exist $m, n \in \mathbb{Z}$ such that

$$
\begin{aligned}
\frac{m-1}{s} \leq & a<\frac{m}{s} \leq \frac{n}{s}<b \leq \frac{n+1}{s} \\
& \Longrightarrow \#\left\{\frac{t}{s} \mid t \in \mathbb{Z}, \frac{t}{s} \in(a, b)\right\}=n-m+1>(b-a) s-1 .
\end{aligned}
$$

In particular, if $q_{o} \in S, \Pi_{\alpha}\left(z_{o}\right)=q_{o}$ and $I=\Pi_{\alpha}\left(z_{o}+(a, b)\right)$, then

(7) $N\left(\alpha, q_{o}, I\right)=\#\left[\left(z_{o}+\Gamma_{\mathbb{R}}(\alpha) \bmod (1)\right) \cap\left(z_{o}+(a, b) \bmod (1)\right)\right]$

$$
>(b-a) s-1 .
$$

Fix $\alpha_{o} \in \mathbb{R} \backslash \mathbb{Q}$ and sequences $\left(\alpha_{n}\right)_{n>1}$ in $\mathbb{Q}$ and $\left(q_{n}\right)_{n>1}$ in $S$, where $\lim _{n \rightarrow \infty} \alpha_{n}=\alpha_{o}$. Since $\alpha_{o} \notin \mathbb{Q}$, we can write $\mu\left(\alpha_{n}-1\right)=r_{n} / s_{n}$, where $r_{n}, s_{n} \in \mathbb{Z}, \operatorname{gcd}\left(r_{n}, s_{n}\right)=1$ and $\lim _{n \rightarrow \infty} s_{n}=+\infty$. It follows from (7) that

$$
N\left(\alpha_{n}, q_{n}, I\right)>(b-a) s_{n}-1 \Longrightarrow \lim _{n \rightarrow \infty} N\left(\alpha_{n}, q_{n}, I\right)=+\infty .
$$

\section{Pulling-back the family $\mathbb{F}_{4}$}

In the process of pulling-back the pencil $\mathbb{F}_{4}$ we will consider a polynomial map, depending on $\alpha \in \mathbb{R}, \Phi_{\alpha}: \mathbb{R} \mathbb{P}(2) \rightarrow \mathbb{R} \mathbb{P}(2)$ with the following properties:

(i) There are algebraic curves $F, F_{\alpha} \subset \mathbb{R} \mathbb{P}(2)$ and $p_{o} \in F, q_{o} \in F_{\alpha}$ such that $\Phi_{\alpha}(F)=F_{\alpha}$ and $\Phi_{\alpha}\left(p_{o}\right)=q_{o}$.

(ii) There are local coordinates $\left(U,(u, v) \in \mathbb{R}^{2}\right)$ and $\left(U_{\alpha},(x, y) \in \mathbb{R}^{2}\right)$ such that $u\left(p_{o}\right)=v\left(p_{o}\right)=0, x\left(q_{o}\right)=y\left(q_{o}\right)=0, F \cap U=(v=0)$, $F_{\alpha} \cap U_{\alpha}=(y=0)$ and $\Phi_{\alpha}(u, v)=\left(u, v^{2}\right)=(x, y)$. In other words, $\Phi_{\alpha}$ folds around $F$ in the sense of Whitney.

Let us suppose that the point $q_{o}$ is not contained in the set $\mathcal{L}$. Since $\mathcal{F}_{0}^{4}$ and $\mathcal{F}_{\infty}^{4}$ are transverse outside $\mathcal{L}$, there is an unique $\alpha \in \mathbb{R} \cup\{\infty\}$ such that the leaf of $\mathcal{F}_{\alpha}^{4}$ through $q_{o}$ is tangent to $F_{\alpha}$ at $q_{o}$. This condition implies that:

(iii) The foliation $\mathcal{F}_{\alpha}^{4}$ can be defined in $\left(U_{\alpha},(x, y)\right)$ by a differential equation of the form $d y-Q(x, y) d x=0$, where $Q(0,0)=0$.

Let us assume further that $Q_{x}(0,0)=-a<0$. The pull-back foliation $\mathcal{F}_{\alpha}:=\Phi^{*}\left(\mathcal{F}_{\alpha}^{4}\right)$ is defined in the chart $(U,(u, v))$ by $\mu=0$, where $\mu=$ $2 v d v-Q\left(u, v^{2}\right) d u$, or by the vector field $X=2 v \partial_{u}+Q\left(u, v^{2}\right) \partial_{v}$. The eigenvalues of $D X(0,0)$ are $\pm i \sqrt{2 a}$ and the singularity $p_{o}=(0,0)$ is a center for $\mathcal{F}_{\alpha}$. 

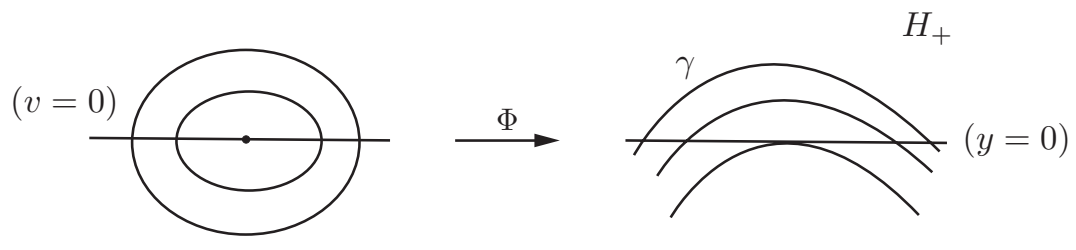

Figure 1

In fact, with the condition $Q_{x}(0,0)<0$, the real leaf of $\mathcal{F}_{\alpha}^{4}$ through $q_{o}$ has a tangency of order one with the line $(y=0)$, as shown in Figure 1. A nearby leaf of $\left.\mathcal{F}_{\alpha}^{4}\right|_{U_{1}}$ in the set $H_{+}:=(y \geq 0)$, say $\gamma$, cuts the line $(y=0)$ in two points and $\gamma \cap H_{+}$is a segment. In this case, $\Phi^{-1}\left(\gamma \cap H_{+}\right)$is a closed curve in the plane $(u, v)$. Hence, $p_{o}$ is a center for $\mathcal{F}_{\alpha}$.

Let us specify the map $\Phi_{\alpha}$. Choose the point $q_{o} \in \mathbb{R P}(2)$ as $q_{o}=\pi\left(q_{1}\right)$, where $q_{1} \in S$. Note that $q_{o} \notin \mathcal{L}$. Let $(x, y) \in \mathbb{R}^{2} \subset \mathbb{C}^{2}$ be the affine coordinates system fixed in the introduction and $q_{o}=\left(a_{o}, b_{o}\right)$ in this coordinates system. Denote by $L_{\alpha}$ the straight line tangent to the leaf $\ell_{\alpha}$ of $\mathcal{F}_{\alpha}^{4}$ through $q_{o}$. The slope of $L_{\alpha}$ at $q_{o}$ is

$$
\phi(\alpha):=\frac{\left(b_{o}^{3}-1\right)\left(b_{o}-\alpha \cdot a_{o}^{2}\right)}{\left(a_{o}^{3}-1\right)\left(a_{o}-\alpha \cdot b_{o}^{2}\right)} .
$$

If we choose $\alpha \neq a_{o} / b_{o}^{2}$ then $L_{\alpha}$ is not vertical and can be parametrized as $s \mapsto\left(s+a_{o}, \phi(\alpha) . s+b_{o}\right)$.

Set

$$
\Phi_{a}(s, t)=\left(s+a_{o}, \pm t^{2}+\phi(\alpha) \cdot s+b_{o}\right)=(x, y) .
$$

Note that $\Phi_{\alpha}(0,0)=q_{o}$ and $\Phi_{\alpha}(t=0)=L_{\alpha}$. From now on, we will denote by $\mathbb{R}_{(s, t)}^{2}$ the domain of $\Phi_{\alpha}$.

If the sign in the second component of $(8)$ is + then

$$
\Phi_{\alpha}\left(\mathbb{R}_{(s, t)}^{2}\right)=\left\{(x, y) \mid y \geq \phi(\alpha) \cdot\left(x-a_{o}\right)+b_{o}\right\}:=H_{+}(\alpha),
$$

whereas if the sign is - then

$$
\Phi_{\alpha}\left(\mathbb{R}_{(s, t)}^{2}\right)=\left\{(x, y) \mid y \leq \phi(\alpha) .\left(x-a_{o}\right)+b_{o}\right\}:=H_{-}(\alpha) .
$$

In particular, $\Phi_{\alpha}$ folds $\mathbb{R}_{(s, t)}^{2}$ around the line $(t=0)$. 
We have to impose an additional condition on $\alpha$ to guarantee that the tangency of $\ell_{\alpha}$ with $L_{\alpha}$ at $q_{o}$ is of order one. The set of points $p \in \mathbb{C P}(2)$ where the tangency of the leaf of some foliation $\mathcal{G}$ through $p$ with its tangent line at $p$ is of order greater than one, called the inflection divisor of $\mathcal{G}$, was computed in $[\mathbf{J P}]$. Applying this computation in the case of $\mathcal{F}_{\alpha}^{4}$ we find the following equation for its inflection divisor:

$$
P(x, y, \alpha)=\left(y^{3}-1\right)\left(x^{3}-1\right)\left(x^{3}-y^{3}\right)\left(\left[2+\alpha^{3}\right] x y-\alpha x^{3}-\alpha y^{3}-\alpha\right) .
$$

Since $q_{o}=\left(a_{o}, b_{o}\right) \notin \mathcal{L}=\left(\left(x^{3}-1\right)\left(y^{3}-1\right)\left(y^{3}-x^{3}\right)=0\right)$ we have to choose $\alpha$ in such a way that $\left[2+\alpha^{3}\right] a_{o} b_{o}-\alpha a_{o}^{3}-\alpha b_{o}^{3}-\alpha \neq 0$.

The sign of $\pm t^{2}$ in (8) is choosen to be + if the leaf $\ell_{\alpha}$ (near $q_{o}$ ) is contained in the region $H_{-}(\alpha)$ and - otherwise. Note that in the first case $\Phi_{\alpha}\left(\mathbb{R}_{(s, t)}^{2}\right) \subset H_{+}(\alpha)$ and in the second $\Phi_{a}\left(\mathbb{R}_{(s, t)}^{2}\right) \subset H_{-}(\alpha)$. With these conditions, $p_{o}=(0,0)$ is a center for the real pull-back foliation

$$
\mathcal{F}_{\alpha}:=\Phi_{\alpha}^{*}\left(\mathcal{F}_{\alpha}^{4}\right)
$$

Fix $\alpha_{o} \in \mathbb{R}$ satisfying the above conditions and assume that $\ell_{\alpha_{o}}$ near $q_{o}$ is contained in the region $H_{-}\left(\alpha_{o}\right)$, so that we choose the sign + in (8) for $\Phi_{\alpha_{o}}$.

From continuity and the arguments already exposed there exist $0<$ $\epsilon \leq \epsilon_{1}, \delta, \delta_{1}>0$ such that if $\alpha \in J:=\left(\alpha_{o}-\epsilon, \alpha_{o}+\epsilon\right)$ then:

(iv) If $p_{o}=(0,0)$ then $\Phi_{\alpha}\left(p_{o}\right)=q_{o}$ and $p_{o}$ is a center for $\mathcal{F}_{\alpha}$ (see Figure 1).

(v) If $K:=(-\delta,+\delta) \times\{0\}$ then the segment $\Phi_{\alpha}(K) \subset L_{\alpha}$ contains a segment $I_{\alpha}$ of euclidean lenght $2 \delta_{1}$ of the line $L_{\alpha}$, centered in $q_{o}$.

(vi) $\mathcal{F}_{\alpha}^{4}$ is transverse to $L_{\alpha}$ in all points of $I_{\alpha} \backslash\left\{q_{o}\right\}$.

Let $D \subset \mathbb{R}^{2}$ be the disk of radius $\delta_{1}$ centered at $q_{o}$. Denote $D_{+}^{\alpha}:=$ $H_{+}(\alpha) \cap D$. Recall that $\pi(S)$ is the real leaf of $\mathcal{F}_{\infty}^{4}$ through $q_{o}$. Since $\mathcal{F}_{\infty}^{4}$ and $\mathcal{F}_{\alpha}^{4}$ are transverse at $q_{o}$ we can choose a segment $I$ contained in $S$ with the following properties:

(vii) $q_{o}$ is in one of the extremities of $\pi(I)$ and $\pi(I) \subset D_{+}(\alpha)$.

(viii) For all $q \in \pi(I)$ and $\alpha \in J$ the leaf $\ell_{q}$ of $\left.\mathcal{F}_{\alpha}^{4}\right|_{D}$ cuts $I_{\alpha}$ in two points, say $q_{+}$and $q_{-}$, one in each side of $q_{o}$ in $I_{\alpha}$.

The situation described above is sketched in Figure 2. 


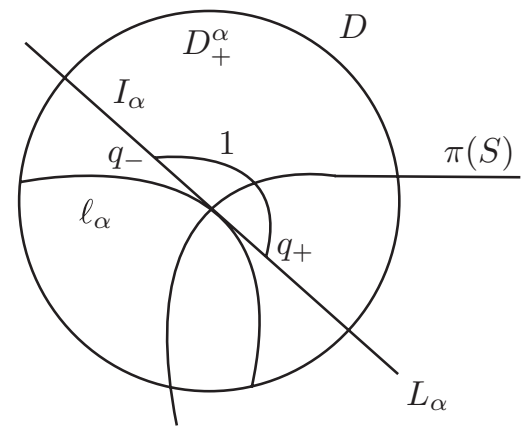

Figure 2

Let $I_{1}(\alpha) \subset \mathbb{R}_{(s, t)}^{2}$ be the connected component of $\Phi_{\alpha}^{-1}(\pi(I))$ which contains $p_{o}=0$. Note that $I_{1}(\alpha)$ is a segment of curve in $\mathbb{R}^{2}$, because $\pi(I)$ is transverse to $L_{\alpha}$. Moreover, if $h_{\alpha}:=\left.\Phi_{\alpha}\right|_{I_{1}(\alpha)}: I_{1}(\alpha) \rightarrow \pi(I)$ then $h_{\alpha}^{-1}\left(q_{o}\right)=p_{o}$ and $\#\left(h_{\alpha}^{-1}(q)\right)=2$ if $q \in I_{1}(\alpha) \backslash\left\{q_{o}\right\}$, because $\Phi_{\alpha}$ folds at the line $(t=0)$ and $\Phi_{\alpha}\left(\mathbb{R}_{(s, t)}^{2}\right)=H_{+}(\alpha)$.

(ix) Given $p \in I_{1}(\alpha) \backslash\left\{p_{o}\right\}$ the real leaf of $\mathcal{F}_{\alpha}$ through $p$ is a closed curve (diffeomorphic to $\mathbb{S}^{1}$ ).

In fact, $h_{\alpha}(p)=q \in I$ and the leaf of $\mathcal{F}_{\alpha}^{4}$ through $q$ cuts $I_{\alpha}$ in two points $q_{-}$and $q_{+}$, determining in this way a segment 1 between these points, as shown in Figure 2. Since $\mathcal{F}_{\alpha}=\Phi_{\alpha}^{*}\left(\mathcal{F}_{\alpha}^{4}\right)$ the leaf of $\mathcal{F}_{\alpha}$ through $p$ is $\Phi_{\alpha}^{-1}(\mathrm{l})$ which is a closed curve (see Figure 1 ).

Let us prove that the family $\left(\mathcal{F}_{\alpha}\right)_{\alpha \in J}$ satisifies properties (P.1), (P.2) and (P.3) in the statement of Theorem 1. Property (P.1) follows from the fact that $E_{4} \cap \mathbb{R}=\mathbb{Q}$, where $E_{4}=\left\{\alpha \in \mathbb{C} \mid \mathcal{F}_{\alpha}^{4}\right.$ has a non-constant rational first integral\}.

In order to prove that it satisfies (P.2) and (P.3) we will consider a slightly more general situation. Let $M$ and $N$ be two complex compact surfaces and $\Psi: M \rightarrow N$ be a non-degenerate rational map of topological degree $\operatorname{dg}(\Psi)=k \geq 2$. Let $C P(\Psi) \subset M$ be the set of critical points of $\Psi$ and $C V(\Psi)=\Psi(C P(\Psi))$ be its set of critical values. The fact that $\operatorname{dg}(\Psi)=k$ means that for any $q \notin C V(\Psi)$ we have $\#\left(\Psi^{-1}(q)\right)=k$. Recall also that both sets $C P(\Psi)$ and $C V(\Psi)$ are holomorphic curves. Let $\mathcal{G}$ be a holomorphic foliation on $M$ and set $\mathcal{G}^{*}:=\Psi^{*}(\mathcal{G})$.

Lemma 3.1. In the above situation, given a leaf $L$ of $\mathcal{G}$ not contained in $C V(\Psi)$, define $L^{*}$ as the satured set of $\Psi^{-1}(L \backslash C V(\Psi))$ by the foliation $\mathcal{G}^{*}$. Then $L^{*}$ is an union at most $k$ leaves of $\mathcal{G}^{*}$. 
Proof: In fact, since $\Psi$ is non-degenerate, $\Psi^{-1}(C V(\Psi))$ is a holomorphic curve in $M$. Let $X:=M \backslash \Psi^{-1}(C V(\Psi))$ and $Y:=N \backslash C V(\Psi)$. It is known that $X$ and $Y$ are open and dense in $M$ and $N$, respectively. Moreover, $\psi:=\left.\Psi\right|_{X}: X \rightarrow Y$ is a holomorphic covering map with $k$-sheets. Let $\mathcal{G}_{Y}:=\left.\mathcal{G}\right|_{Y}$ and $\mathcal{G}_{X}^{*}:=\left.\mathcal{G}^{*}\right|_{X}$. Since $L$ is a leaf of $\mathcal{G}$ not contained in $C V(\Psi)$, which is a curve, $L \cap C V(\Psi)$ is a discrete subset of $L$ in its intrinsic topology (cf. [C-LN]). Therefore, $L_{Y}:=L \cap Y$ is connected, which implies that it is a leaf of $\mathcal{G}_{Y}$. Set $L_{X}:=\psi^{-1}\left(L_{Y}\right)$ and $\psi_{L}:=\left.\psi\right|_{L_{X}}: L_{X} \rightarrow L_{Y}$. Note that $L_{X}$ is an union of leaves of $\mathcal{G}_{X}^{*}$, because $\psi$ is a local biholomorphism. If we consider these leaves with the intrinsic topology, the map $\psi_{L}$ is a covering map with $k$-sheets. This implies that $L_{X}$ has at most $k$ connected components, so that it is an union of at most $k$ leaves of $\mathcal{G}_{X}^{*}$. This implies that $L^{*}$ is an union of at most $k$ leaves of $\mathcal{G}^{*}$.

Denote by $\Phi_{\mathbb{C}, \alpha}$ and $\mathcal{F}_{\mathbb{C}, \alpha}$ the complexifications of $\Phi_{\alpha}$ and $\mathcal{F}_{\alpha}$, respectively. With these notations we have $\mathcal{F}_{\mathbb{C}, \alpha}=\Phi_{\mathbb{C}, \alpha}^{*}\left(\mathcal{F}_{\alpha}^{4}\right)$. Note that $\Phi_{\mathbb{C}, \alpha}$ can be considered as a rational map $\mathbb{C P}(2) \rightarrow \mathbb{C P}(2)$ of topological degree two. Moreover, $C P\left(\Phi_{\mathbb{C}, \alpha}\right) \cap \mathbb{C}^{2}=(t=0)$ and $C V\left(\Phi_{\mathbb{C}, \alpha}\right) \cap \mathbb{C}^{2}=L_{\alpha}$. Given $q \in \mathbb{R}^{2} \backslash \operatorname{sing}\left(\mathcal{F}_{\alpha}^{4}\right)$ denote the complex leaf of $\mathcal{F}_{\alpha}^{4}$ through $q$ by $\ell(q, \alpha)$. Following the notation of Lemma 3.1, let $\ell^{*}(q, \alpha)$ be the satured set of $\Phi_{\mathbb{C}, \alpha}^{-1}\left(\ell(q, \alpha) \backslash C V\left(\Phi_{\mathbb{C}, \alpha}\right)\right.$ by the foliation $\mathcal{F}_{\mathbb{C}, \alpha}$. According to Lemma $3.1, \ell^{*}(q, \alpha)$ contains at most two leaves of $\mathcal{F}_{\mathbb{C}, \alpha}$.

Remark 3.1. If $\ell(q, \alpha)$ is transverse to $L_{\alpha}$ at some point of $\ell(q, \alpha) \cap L_{\alpha} \cap \mathbb{C}^{2}$ then $\ell^{*}(q, \alpha)$ is a leaf of $\mathcal{F}_{\mathbb{C}, \alpha}$.

Proof: Assume that $\ell^{*}(q, \alpha)$ contains two different leaves of $\mathcal{F}_{\mathbb{C}, \alpha}$, say $\ell_{1}$ and $\ell_{2}$. In this case, if $m \in \ell(q, \alpha) \backslash L_{\alpha}$ then $\Phi_{\mathbb{C}, \alpha}^{-1}(m)$ contains two points, one in $\ell_{1}$ and the other in $\ell_{2}$. Let $m_{o} \in L_{\alpha} \cap \ell(q, \alpha) \cap \mathbb{C}^{2}$ and suppose by contradiction that $\ell(q, \alpha)$ is transverse to $L_{\alpha}$ at $m_{o}$. Note that $\Phi_{\mathbb{C}, \alpha}^{-1}\left(m_{o}\right)=\left\{m_{1}\right\}$. It follows from (8) that there are germs of coordinates systems $(u, v)$ and $(z, w)$ such that $u\left(m_{1}\right)=v\left(m_{1}\right)=0$, $z\left(m_{o}\right)=w\left(m_{o}\right)=0, L_{\alpha}=(w=0), \Phi_{\mathbb{C}, \alpha}(u, v)=\left(u, v^{2}\right)=(z, w)$ and $\ell(q, \alpha)$ near $m_{o}$ can be parametrized by $w \mapsto(\psi(w), w), \psi(0)=$ 0 . In this case, the curve $C$ parametrized by $v \mapsto\left(\psi\left(v^{2}\right), v\right)$ satisfies $\Phi_{\mathbb{C}, \alpha}(C) \subset \ell(q, \alpha)$, so that $C \subset \ell^{*}(q, \alpha)$. On the other hand, if $w \neq 0$ and $m=(\psi(w), w)$ then $\Phi_{\mathbb{C}, \alpha}^{-1}(m)$ contains two points in $C$ which implies that $\ell_{1}=\ell_{2}$. Hence, $\ell^{*}(q, \alpha)$ is a leaf of $\mathcal{F}_{\mathbb{C}, \alpha}$.

As a consequence of (ix) and of Remark 3.1 we obtain the following: 
(x) With the notations of Remark 3.1, assume that $\ell(q, \alpha)$ is transverse to $L_{\alpha}$ at some point of $L_{\alpha} \cap \ell(q, \alpha)$. Then for each point $q_{1} \in$ $\pi(I) \cap \ell(q, \alpha)$ the real foliation $\mathcal{F}_{\alpha}$ has a closed leaf contained in $\ell^{*}(q, \alpha) \cap \mathbb{R P}(2)$.

For fixed $\lambda \in J \cap \mathbb{Q}$ and $p \in I_{1}(\lambda) \backslash\left\{p_{o}\right\}$, denote by $F_{\lambda}$ a primitive real rational first integral of $\mathcal{F}_{\lambda}$ and by $O(\lambda, p)$ the number of real connected components of $F_{\lambda}^{-1}\left(F_{\lambda}(p)\right)$. Let $F_{\mathbb{C}, \lambda}$ be the complexification of $F_{\lambda}$. Note that the leaf of $\mathcal{F}_{\mathbb{C}, \lambda}$ through $p$ is

$$
\tilde{\ell}(p):=F_{\mathbb{C}, \lambda}^{-1}\left(F_{\lambda}(p)\right) \backslash \operatorname{sing}\left(\mathcal{F}_{\mathbb{C}, \alpha}\right) .
$$

It follows from Remark 3.1 that $\tilde{\ell}(p)=\ell^{*}(q, \lambda)$, where $q=\Phi_{\alpha}(p)$, because $\ell(q, \lambda)$ cuts transversely $L_{\alpha}$ at $q_{+}$and $q_{-}$(see Figure 2). Let $\hat{q} \in I \subset S$ be such that $\pi(\hat{q})=q$. If $N(\lambda, \tilde{q}, I)$ is like in Lemma 2.2 then we get from $(\mathrm{x})$ that

$$
O(\lambda, p) \geq N(\lambda, \hat{q}, I) .
$$

Therefore, (b) of Lemma 2.2 implies that the family $\left(\mathcal{F}_{\alpha}\right)_{\alpha \in J}$ satisfies property (P.2). With the same type of argument, it is possible to prove that (a) of Lemma 2.2 implies that it satisfies property (P.3). We leave the details for the reader.

Finally, the family $\left(\mathcal{F}_{\alpha}\right)_{\alpha \in J}$ is in $\operatorname{Fol}_{\mathbb{R}}(2,8)$ because $\Phi_{\alpha}^{*}\left(\omega-\alpha \cdot \omega_{\infty}\right)=$ $P_{\alpha}(s, t) d t-Q_{\alpha}(s, t) d s$, where $P_{\alpha}$ and $Q_{\alpha}$ have degree 8 and the homogeneous term of degree eight of $t . P_{\alpha}(s, t)-s . Q_{\alpha}(s, t)$ is not identically zero, as the reader can check.

\section{Other families}

In this section we will describe briefly how to obtain families of foliations of any degree $\geq 5$ satisfying properties (P.1), (P.2) and (P.3) of Theorem 1. These families will be obtained by pulling-back the family $\mathbb{F}_{2}:=\left(\mathcal{F}_{\alpha}^{2}\right)_{\alpha \in \mathbb{R}}$ already mentioned in Section 1 . The foliation $\mathcal{F}_{\alpha}^{2}$ is defined by the differential equation $\omega_{\alpha}:=\omega-\alpha \cdot \omega_{\infty}=0$, where

$$
\left\{\begin{array}{l}
\omega=\left(4 x-9 x^{2}+y^{2}\right) d y-(6 y-12 x y) d x \\
\omega_{\infty}=(2 y-4 x y) d y-3\left(x^{2}-y^{2}\right) d x
\end{array} .\right.
$$

We would like to remark that the set $\operatorname{Tang}\left(\mathcal{F}_{0}^{2}, \mathcal{F}_{\infty}^{2}\right)$ consists of two irreducible curves: the line at infinity of $\mathbb{C}^{2}, L_{\infty}$, and the quartic $Q=$ $(P=0)$, where $P(x, y)=4 y^{2}(1-3 x)-4 x^{3}+\left(3 x^{2}+y^{2}\right)^{2}$. These two curves 
are invariant for all foliations in the pencil. Moreover, $\operatorname{sing}\left(\mathcal{F}_{\alpha}^{2}\right) \subset Q \cup L_{\infty}$ for any $\alpha \in \overline{\mathbb{C}}$.

The connection between the familes $\mathbb{F}_{2}$ and $\mathbb{F}_{4}$ is that there exists a rational map of topological degree two $\Psi: \mathbb{C P}(2) \rightarrow \mathbb{C P}(2)$ such that $\Psi^{*}\left(\mathcal{F}_{\alpha}^{2}\right)=\mathcal{F}_{\alpha}^{4}$ for all $\alpha \in \overline{\mathbb{C}}$. This map satisfies $\Psi(\mathbb{R} \mathbb{P}(2))=\mathbb{R} \mathbb{P}(2)$ (cf. $[\mathbf{L N}]$ ). As a consequence, the set $E_{2}=\left\{\alpha \in \overline{\mathbb{C}} \mid \mathcal{F}_{\alpha}^{2}\right.$ has a nonconstant rational first integral $\}$ coincides with $E_{4}=\mathbb{Q} . \Gamma \cup\{\infty\}$. Moreover, a statement analogous to Lemma 2.2 is true.

Let us precise the last assertion, but before that, we will fix some notations. In order to avoid confusion, when $\alpha \in \mathbb{R} \cup\{\infty\}$ we will denote by $\mathcal{F}_{\mathbb{R}, \alpha}^{2}$ the real foliation induced by $\omega_{\alpha}$ in $\mathbb{R P}(2)$. Given $q \in \mathbb{R} \mathbb{P}(2)$ denote by $\ell(q, \alpha)$ the (complex) leaf of $\mathcal{F}_{\alpha}^{2}$ through $q$. Set

$$
\ell_{\mathbb{R}}(q, \alpha)=\mathbb{R} \mathbb{P}(2) \cap \ell(q, \alpha) .
$$

We would like to remark that $\ell_{\mathbb{R}}(q, \alpha)$ is an union of leaves of $\mathcal{F}_{\mathbb{R}, \alpha}^{2}$. Each leaf of $\mathcal{F}_{\mathbb{R}, \alpha}^{2}$ is homeomorphic to $\mathbb{R}$ and accumulates in at most two points of $\operatorname{sing}\left(\mathcal{F}_{\mathbb{R}, \alpha}^{2}\right)$ : the foliation has no closed leaf (homeomorphic to $\left.\mathbb{S}^{1}\right)$. When $\alpha \in \mathbb{R} \backslash \mathbb{Q}$ and $q \in \mathbb{R} \mathbb{P}(2) \backslash\left(Q \cup L_{\infty}\right)$ then $\ell_{\mathbb{R}}(q, \alpha)$ has an infinite number of connected components, whereas if $\alpha \in \mathbb{Q} \cup\{\infty\}$ then it has a finite number.

In the case of $\mathcal{F}_{\infty}^{2}$, we have the first integral $G_{\infty}(x, y):=P(x, y) /(2 x-$ $1)^{3}$ (cf. [LN-1]). In particular, given $q_{o} \in \mathbb{R P}(2) \backslash\left(Q \cup L_{\infty}\right)$ then the closure of $\ell_{\mathbb{R}}\left(q_{o}, \infty\right)$ is precisely $G_{\infty}^{-1}\left(G_{\infty}\left(q_{o}\right)\right) \cap \mathbb{R} \mathbb{P}(2)$. Moreover, if $q_{1} \in \mathbb{R P}(2)$ is such that $\Psi\left(q_{1}\right)=q_{o}$ then $G_{\infty}^{-1}\left(G_{\infty}\left(q_{o}\right)\right)=\Psi\left(\pi\left(S_{c}\right)\right)$, for some $c \in \mathbb{R}$, where $q_{1} \in S_{c}$ and $S_{c}$ is like in Section 2. From now on, we will fix $q_{o} \in \mathbb{R P}(2) \backslash\left(Q \cup L_{\infty}\right)$ and set $S_{1}:=\ell_{\mathbb{R}}\left(q_{o}, \infty\right)$.

An interval $I \subset S_{1}$ will be by convention the image of some interval $I_{1} \subset S_{c}: I=\Psi\left(\pi\left(I_{1}\right)\right)$. Given $q \in S_{1} \backslash\left(Q \cap L_{\infty}\right)$ set

$$
N(\alpha, q, I)=\#\left[I \cap \ell_{\mathbb{R}}(\alpha, q)\right] .
$$

If $G_{\alpha}$ is a real primitive first integral of $\mathcal{F}_{\mathbb{R}, \alpha}^{2}$ then

$$
N(\alpha, q, I)=\#\left[I \cap G_{\alpha}^{-1}\left(G_{\alpha}(q)\right)\right] .
$$

We have the following consequence of Lemma 2.2:

Corollary 4.1. With the above notations, we have:

(a) If $q \in S_{1}$ then $\ell_{\mathbb{R}}(\alpha, q) \cap S_{1}$ is dense in $S_{1} \Longleftrightarrow \alpha \in \mathbb{R} \backslash \mathbb{Q}$.

(b) Let $\alpha_{o} \in \mathbb{R} \backslash \mathbb{Q}$ and $\left(\alpha_{n}\right)_{n \geq 1}$ be a sequence in $\mathbb{Q}$ such that $\lim _{n \rightarrow \infty} \alpha_{n}=$ $\alpha_{o}$. Then for any non-trivial segment $I$ of $S_{1}$ and any sequence 
$\left(q_{n}\right)_{n \geq 1}$ in $S_{1}$ we have

$$
\lim _{n \rightarrow \infty} N\left(\alpha_{n}, q_{n}, I\right)=+\infty
$$

It follows that we can apply to the family $\mathbb{F}_{2}$ the same method of Section 3 to obtain families satisfying properties (P.1), (P.2) and (P.3) of Theorem 1. The inflexion divisor of $\mathcal{F}_{\alpha}^{2}$ is a curve of degree six in $\mathbb{C P}(2)$ which contains the line $L_{\infty}$. Its intersection with $\mathbb{R}^{2}=\mathbb{R P}(2) \backslash L_{\infty}$ is an irreducible curve $C_{\alpha}$ of degree five. Given $q=(a, b) \in \mathbb{R}^{2}$ denote by $L_{\alpha}(q)$ the line tangent to $\mathcal{F}_{\alpha}$ at $q$. The slope of $L_{\alpha}(q)$ is

$$
\phi(q, \alpha)=\frac{6 b-12 a b-3 \alpha\left(a^{2}-b^{2}\right)}{4 a-9 a^{2}+b^{2}-\alpha(2 b-4 a b)} .
$$

It is clear that we can choose $q_{o}=\left(a_{o}, b_{o}\right) \in \mathbb{R}^{2}$ and $\alpha_{o}$ in such a way that $\phi\left(q,{ }_{o}, \alpha_{o}\right) \neq \infty$ and $q_{o} \notin C_{\alpha_{o}}$. Let $\epsilon>0$ be such that $\phi(\alpha):=\phi\left(q_{o}, \alpha\right) \neq$ $\infty$ and $q_{o} \notin C_{\alpha}$ for all $\alpha \in\left(\alpha_{o}-\epsilon, \alpha_{o}+\epsilon\right):=J$. The line $L_{\alpha}$ is not vertical and can be parametrized by $s \mapsto\left(s+a_{o}, \phi(\alpha) . s+b_{o}\right)$. When we pull-back $\left(\mathbb{F}_{\alpha}^{2}\right)_{\alpha \in J}$ by the family of maps $\left(\Phi_{\alpha}\right)_{\alpha \in J}$ given in (8) we obtain a family of foliations $\left(\mathcal{F}_{\alpha}\right)_{\alpha \in J}$ of degree five. In fact, if we set $\omega_{\alpha}=$ $P_{\alpha}(x, y) d y-Q_{\alpha}(x, y) d x$, where $P_{\alpha}(x, y)=4 x-9 x^{2}+y^{2}-\alpha(2 y-4 x y)$ and $Q_{\alpha}(x, y)=6 y-12 x y-3 \alpha\left(x^{2}-y^{2}\right)$, then

$$
\begin{aligned}
\Phi_{\alpha}^{*}\left(\omega_{\alpha}\right)=P_{\alpha} \circ \Phi_{\alpha}(s, t)( \pm t d t+\phi(\alpha) \cdot d s) & -Q_{\alpha} \circ \Phi_{\alpha}(s, t) d s \\
& :=\hat{P}_{\alpha}(s, t) d t-\hat{Q}_{\alpha}(s, t) d s .
\end{aligned}
$$

Since $\hat{P}_{\alpha} \circ \Phi_{a}(s, t)$ has degree 5 and $\hat{Q}_{\alpha} \circ \Phi_{\alpha}(s, t)$ degree $\leq 4$, the foliation $\mathcal{G}_{\alpha}:=\Phi_{\alpha}^{*}\left(\mathcal{F}_{\alpha}^{2}\right)$ has degree five. We then choose the sign + or - , as indicated in Section 3, in such a way that $\mathcal{G}_{\alpha}$ has a real center at the point $p_{o}=(0,0)\left(\Phi_{\alpha}\left(p_{o}\right)=q_{o}\right)$. In this way, we get a family of degree 5 satisfying (P.1), (P.2) and (P.3) of Theorem 1.

Based in the same idea, we can obtain families of any degree $k \geq 5$ as follows. Consider $\Phi_{\alpha}: \mathbb{R P}(2) \rightarrow \mathbb{R P}(2)$ defined in $\mathbb{R}^{2} \subset \mathbb{R P}(2)$ by

$$
\Phi_{\alpha}(s, t):=\left(s+a_{o}, q(s) \cdot p(t)+\phi(\alpha) \cdot s+b_{o}\right),
$$

where $p(t)=\sum_{j=2}^{d} t^{j}(\operatorname{dg}(p)=d)$ and $q \in \mathbb{R}[s]$ is a polynomial of degree $k \geq 0$ such that $q(0) \neq 0$.

Let $\mathcal{G}_{\alpha}=\Phi_{\alpha}^{*}\left(\mathcal{F}_{\alpha}^{2}\right), \alpha \in J=\left(\alpha_{o}-\epsilon, \alpha_{o}+\epsilon\right)$. The reader can check that $\Phi_{\alpha}^{*}\left(\omega_{\alpha}\right)=\hat{P}_{\alpha}(s, t) d t-\hat{Q}_{\alpha}(s, t) d s$, where

$$
\left\{\begin{array}{l}
\hat{P}_{\alpha}(s, t)=q(s) \cdot p^{\prime}(t) \cdot P_{\alpha} \circ \Phi_{\alpha}(s, t) \\
\hat{Q}_{\alpha}(s, t)=Q_{\alpha} \circ \Phi_{\alpha}(s, t)-\left(q^{\prime}(s) \cdot p(t)+\phi(\alpha)\right) \cdot P_{\alpha} \circ \Phi_{\alpha}(s, t)
\end{array} .\right.
$$


Note that $\operatorname{dg}\left(\hat{P}_{\alpha}(s, t)\right)=3 d+k-1$ and $\operatorname{dg}\left(\hat{Q}_{\alpha}(s, t)\right) \leq 3 d+k-1$. The line at infinity $L$ of the plane $(s, t)$ is invariant for $\mathcal{G}_{\alpha}$. This can be proved by observing that $\Phi_{\alpha}(L)=[0: 1: 0] \in L_{\infty}$, where $L_{\infty}$ is the line at infinity of the plane $(x, y)$, which is invariant for $\mathcal{F}_{\alpha}^{2}$. This implies that the degree of the foliation $\mathcal{G}_{\alpha}$ is $3 d+k-1$. By letting $k \in\{0,1,2\}$ we obtain in this way families of foliations of degrees $3 d-1,3 d, 3 d+1$, $d \geq 2$, and so families of any degree $\geq 5$.

Now, the critical set of $\Phi_{\alpha}$ in $\mathbb{R}^{2}$ is given by $C V\left(\Phi_{\alpha}\right)=\left(q(s) \cdot p^{\prime}(t)=0\right)$ and so it contains the line $(t=0)$. Moreover, $\Phi_{\alpha}(s, 0)=\left(s+a_{o}, \phi(\alpha) . s+\right.$ $\left.b_{o}\right)$ and $\Phi_{\alpha}(t=0)=L_{\alpha}$, the line tangent to the leaf of $\mathcal{F}_{\alpha}^{2}$ through $q_{o}$. On the other hand, if we fix $s=s_{o} \in \mathbb{R}$ such that $q\left(s_{o}\right) \neq 0$, we get

$$
\Phi_{\alpha}\left(s_{o}, t\right)=\left(s_{o}+a_{o}, q\left(s_{o}\right) \cdot p(t)+\phi(\alpha) \cdot s_{o}+b_{o}\right),
$$

which implies that $\Phi_{\alpha}$ sends the line $\left(s=s_{o}\right)$ into the line $\left(x=s_{o}+a_{o}\right)$ folding near $t=0$ because $p(t)=t^{2}+$ h.o.t. Since $q(0) \neq 0$, it follows that there exists a disk $D$ around $0 \in \mathbb{R}^{2}$, which does not depends on $\alpha \in J$, if $\epsilon$ is small enough, such that $\left.\Phi_{\alpha}\right|_{D}$ has a fold line at $(t=0) \cap D$ and $\Phi_{\alpha}(D) \subset H_{+}(\alpha)$ if $q(0)>0$, whereas $\Phi_{\alpha}(D) \subset H_{-}(\alpha)$ if $q(0)<0$. Let $\ell_{\alpha_{o}}$ be the germ at $q_{o}$ of the leaf of $\mathcal{F}_{\alpha_{o}}^{2}$ through $q_{o}$. If $\ell_{\alpha_{o}} \subset H_{-}\left(\alpha_{o}\right)$, as in Figure 2, we choose $q$ so that $q(0)=1$, and if $\ell_{\alpha_{o}} \subset H_{+}\left(\alpha_{o}\right)$ we choose $q(0)=-1$. With this condition, the foliation $\mathcal{G}_{\alpha}$ has a real center at $p_{o}=(0,0)$, as in Figure 1. By applying Corollary 4.1 we obtain families of foliations of any degree $\geq 5$ satisfying properties (P.1), (P.2) and (P.3) of Theorem 1.

\section{References}

[Br] M. BRunella, "Birational geometry of foliations", Monografías de Matemática [Mathematical Monographs], Instituto de Matemática Pura e Aplicada (IMPA), Rio de Janeiro, 2000. Available electronically at http://www.impa.br/Publicacoes/ Monografias/Abstracts/brunella.ps.

[C-LN] C. CAmacho AND A. Lins Neto, "Geometric theory of foliations", Translated from the Portuguese by Sue E. Goodman, Birkhäuser Boston, Inc., Boston, MA, 1985.

[C] M. M. CARnicer, The Poincaré problem in the nondicritical case, Ann. of Math. (2) 140(2) (1994), 289-294.

[Kh] A. G. KhovanskiI,, "Fewnomials", Translated from the Russian by Smilka Zdravkovska, Translations of Mathematical Monographs 88, American Mathematical Society, Providence, RI, 1991. 
[K] K. Kodaira, On compact analytic surfaces. II, III, Ann. of Math. (2) 77 (1963), 563-626; ibid. 78 (1963), 1-40.

[LN] A. Lins Neto, Some examples for the Poincaré and Painlevé problems, Ann. Sci. École Norm. Sup. (4) 35(2) (2002), 231-266.

[LN-1] A. Lins Neto, Curvature of pencils of foliations. Analyse complexe, systèmes dynamiques, sommabilité des séries divergentes et théories galoisiennes. I, Astérisque 296 (2004), 167-190.

[JP] J. V. Pereira, Vector fields, invariant varieties and linear systems, Ann. Inst. Fourier (Grenoble) 51(5) (2001), 1385-1405.

[Se] A. Seidenberg, Reduction of singularities of the differential equation $A d y=B d x$, Amer. J. Math. 90 (1968), 248-269.

Instituto de Matemática Pura e Aplicada

Estrada Dona Castorina, 110

Horto, Rio de Janeiro

Brasil

E-mail address: alcides@impa.br

Primera versió rebuda el 20 de juliol de 2010,

darrera versió rebuda el 19 d'octubre de 2010. 